\title{
N89-25193
}

\section{DIGITAL ROBUST CONTROL LAW SYNTHESIS \\ USING CONSTRAINED OPTIMIZATION}

\author{
Vivekananda Mukhopadhyay \\ Planning Research Corporation \\ MS 243, NASA Langley Research Center \\ Aeroservoelasticity Branch \\ Hampton, Virginia 23665-5225
}

PRECEDING PAGE BLANK NOT FILMED 


\begin{abstract}
Development of digital robust control laws for active control of high performance flexible aircraft and large space structures is a research area of significant practical importance. The flexible system is typically modeled by a large order state space system of equations in order to accurately represent the dynamics. The active control law must satisfy multiple conflicting design requirements and maintain certain stability margins, yet should be simple enough to be implementable on an onboard digital computer. This paper describes an application of a generic digital control law synthesis procedure for such a system, using optimal control theory and constrained optimization technique. A linear quadratic Gaussian type cost function is minimized by updating the free parameters of the digital control law, while trying to satisfy a set of constraints on the design loads, responses and stability margins. Analytical expressions for the gradients of the cost function and the constraints with respect to the control law design variables are used to facilitate rapid numerical convergence. These gradients can be used for sensitivity study and may be integrated into a simultaneous structure and control optimization scheme. An existing control law as well as an estimator based full or reduced order control laws can be optimized in order to meet the multiple design requirements. Low order, robust digital control laws were synthesized for gust load alleviation and flutter suppression of a flexible aircraft.
\end{abstract}




\section{INTRODUCTION}

The small perturbation dynamics of a flexible aircraft or space structure with active control is typically modeled by a large order state space system of equations in order to accurately represent the rigid and flexible body modes, unsteady aerodynamic forces, actuator dynamics, antialiasing filters, computational delays and gust spectra (Ref. 1). The control law of this multiinput multi-output (MIMO) system is expected to satisfy multiple conflicting design requirements on the dynamic loads, root-mean-square (RMS) responses, actuator surface deflection and rate limitations, as well as maintain certain guaranteed stability margins based in the system singular values. Robust control laws for the linear MIMO system with modeling uncertainty can be developed using optimal control theory, which is also known as linear quadratic Gaussian (LQG) technique. This control law is usually of the same or higher order than the plant and is difficult to implement on an onboard digital microprocessor. There are several model reduction techniques to reduce the control law to a lower order but the reduced order control law may not satisfy the design requirements. This paper describes an application of a generic control law synthesis procedure (Ref. 2) for such a system, using optimal control theory and constrained optimization technique. The basic multivariable system and the design problem is schematically described in Fig. 1 . The formulation and synthesis procedure is briefly described first. Application to a gust load alleviation (GLA) of a remotely piloted flexible drone is presented. Some recent results of a flutter suppression system (FSS) design are also presented.

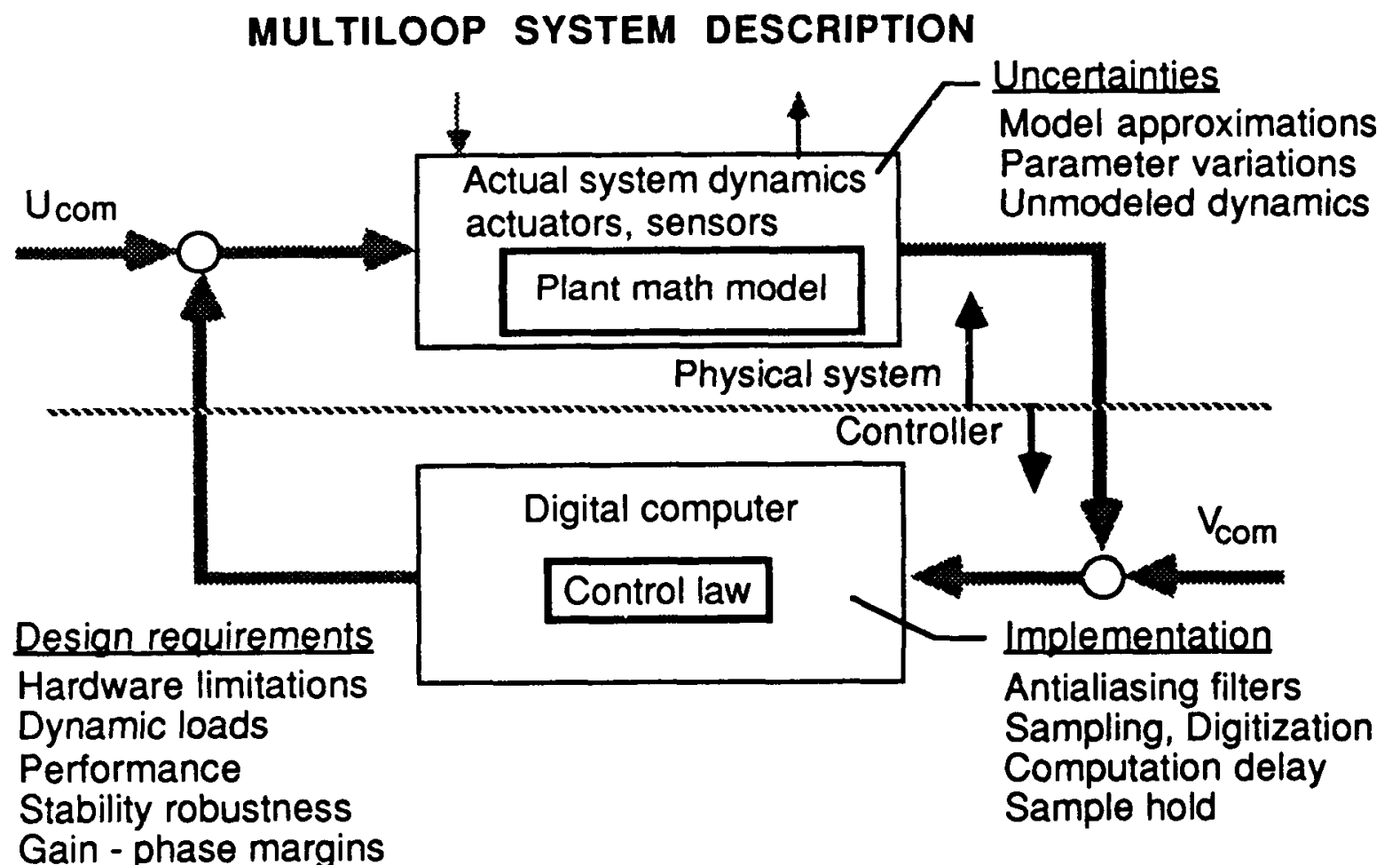

FIGURE 1 


\section{SYSTEM STATE SPACE EQUATIONS}

The typical state space system of equations for a discrete system is shown in Fig. 2. These equations represent discrete time, linear equations of motion, due to a small perturbation from a steady state equilibrium flight condition of a flexible system. The plant equations are usually of large order and include the effects of antialiasing filters and computational delays at each measurement output channel. The antialiasing filters attenuate unmodeled high frequency signals but introduce significant phase lags which must be included in the control law synthesis. The plant and sensor measurement models also contain discrete white noise inputs $\mathbf{w}_{\mathbf{k}}$ and $\mathbf{v}_{\mathbf{k}}$, respectively. The design outputs are the quantities on which design constraints are imposed. The control law is also expressed in state space form and is required to be of lower order than the plant. The discrete control law can be obtained from a full order LQG design after suitable stable order reduction and discretization at a specified sampling rate.

\section{SYSTEM STATE SPACE EQUATIONS}

PLANT

SENSOR

DESIGN

$$
X_{k+1}=F X_{k}+G_{u} u_{k}+G_{w} w_{k}
$$

$$
\mathbf{y}_{\mathbf{k}}=\mathbf{H} \mathbf{X}_{\mathbf{k}}+\mathbf{v}_{\mathbf{k}}
$$

$$
y_{d k}=H_{d} X_{k}+E_{d} u_{k}
$$

$$
\begin{array}{ll}
\text { CONTROL } & Z_{k+1}=A Z_{k}+B y_{k} \\
& u_{k}=C Z_{k}+D y_{k}
\end{array}
$$

FIGURE 2 


\section{AUGMENTED SYSTEM EQUATIONS}

The closed loop system equations can be written in an augmented form as shown in Fig. 3. The new term $\eta$ can be considered as an input command or a fictitious input noise. Using the 'hat' overscript to denote each of the augmented matrices, the closed loop dynamic system looks like a simple output gain feedback system. This type of representation simplifies the derivation of the analytical gradient expressions. Other variations of the augmented system formulation are possible depending upon the controller structure. The design variables are selected parameters of the control law quadruple matrix $\hat{C}$.

\section{CLOSED LOOP AUGMENTED SYSTEM}

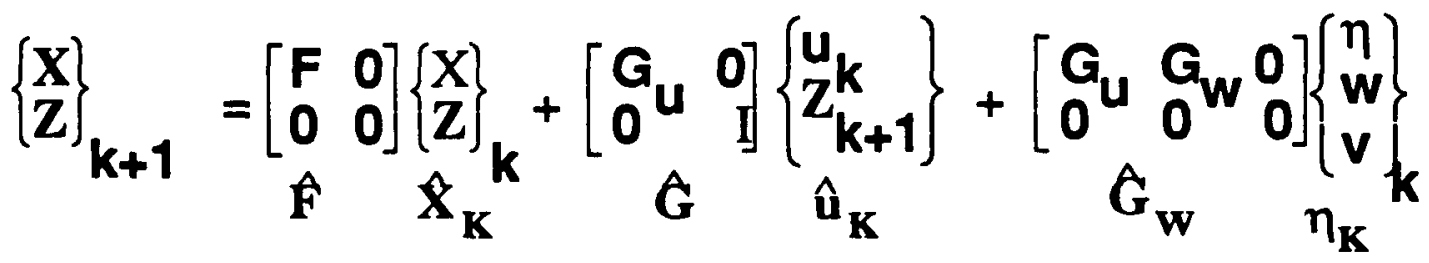

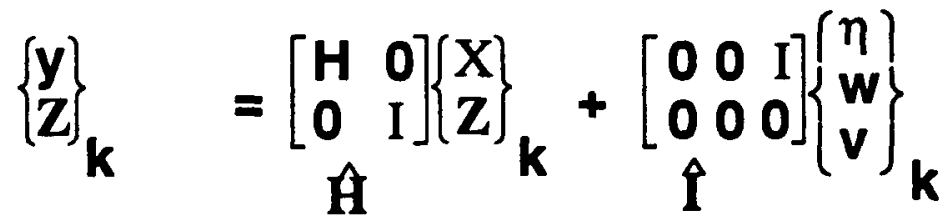

$$
\begin{aligned}
& \left\{\begin{array}{l}
u_{k} \\
z_{k+1}
\end{array}\right\}=\left[\begin{array}{ll}
D & C \\
B & A
\end{array}\right]\left\{\begin{array}{l}
y \\
z
\end{array}\right\}_{k} \\
& \text { C } \hat{\mathbf{y}}_{\mathbf{K}}
\end{aligned}
$$

FIGURE 3 


\section{GRADIENTS}

The analytical expressions for the gradients of the cost function and the constraints with respect to the control law design variables are used for computation. The typical expressions for the gradients are shown in Fig. 4. The underlined matrices are specified for each constraint and cost function. The derivation is quite general in nature. The gradients with respect to other parameters can also be derived in a similar manner. The use of analytical expressions for the gradients in the optimization scheme facilitates rapid convergence of the optimization process. The gradients can also be used for sensitivity study and can be integrated into a simultaneous structure and control optimization scheme. The minimum singular value of the return difference matrix at the plant input and output is also used as additional inequality constraint, in order to improve robustness properties in the frequency domain. These constraints are usually applied at a later stage of the synthesis process.

\section{GRADIENTS OF COST FUNCTION AND CONSTRAINTS}

\section{$d J / d \hat{C}$ and $d g_{n} / d \hat{C}$ are known from steady sta te}

solution of discrete Lyapunov equations

$$
\begin{aligned}
& X_{K+1}=F_{a} X_{K} F_{a}^{\top}+G_{a} R_{a} G_{a}^{\top} \\
& \Lambda_{K}=F_{a}^{\top} \Lambda_{K+1} F_{a}+\underline{Q_{a}}
\end{aligned}
$$

where

$$
\begin{aligned}
& F_{a}=\hat{F}+\hat{G} \hat{C} \hat{H} \\
& G_{a}=\hat{G}_{w}+\hat{G} \hat{C} \hat{i} \\
& \underline{Q}_{a}=\underline{Q}_{1}+(\hat{C} \hat{H})^{T} \underline{Q}_{2}(\hat{C} \hat{H})+(\hat{M} \hat{C} \hat{H})+(\hat{M} \hat{C} \hat{H})
\end{aligned}
$$

FIGURE 4 


\section{PROBLEM DEFFINITION}

The constrained optimization problem is defined as shown in Fig. 5. The control law synthesis procedure minimizes a linear quadratic Gaussian type cost function, subject to a set of constraints on the design loads, RMS responses and stability margins. The stability margin requirement is imposed as constraints on the minimum singular value of the system return difference matrices at the plant input and output (Ref. 3).In a LQG design one has to find a set of weighting matrices and noise intensity matrices in order to satisfy all the RMS response and stability margin requirements. If this trial and error process fails to achieve the desired result, the designer can impose the violated design requirements as RMS response constraints and singular value constraints instead of searching for the weighting matrices.

\section{CONSTRAINED OPTIMIZATION PROBLEM}

$$
\begin{aligned}
& \text { Equations } \hat{x}_{k+1}=\hat{F} \hat{x}_{k}+\hat{G} \hat{u}_{k}+\hat{G}_{w} \hat{\eta}_{k} \\
& \hat{\mathbf{y}}_{k}=\hat{\mathbf{H}} \hat{\mathbf{X}}_{k}+\hat{I} \hat{\eta}_{k} \\
& \hat{u}_{k}=\hat{C} \hat{y}_{k} \\
& \text { Minimize J }=E\left[\left\{\begin{array}{l}
\hat{X} \\
\hat{u}
\end{array}\right\}_{K}^{\top}\left[\begin{array}{ll}
Q & M \\
M^{\top} R
\end{array}\right\}_{\{}\left\{\begin{array}{l}
\hat{X} \\
\hat{u}
\end{array}\right\}_{K S}\right\}_{S S} \\
& \text { By Changing } S[\hat{C}]
\end{aligned}
$$

Subject to inequality constraints on

a) RMS responses and loads

b) Min. singular value

FIGURE 5 


\section{OPTIMIZATION SCHEME}

The constrained optimization problem is solved by using the method of feasible directions (Ref. 4). The optimization scheme block diagram is shown in Fig. 6. This procedure is the discrete time equivalent of those presented in Refs 1,3,5 for a continuous system. Similar procedures without the inequality constraints were also presented in Refs 6-9 in the continuous time domain. The development of the initial stable control law required to start the optimization cycle needs some effort and experience. This is usually done by first designing a full order optimal control law using a continuous plant model, which includes the effects of antialiasing filters and computational delays. The order of the control law is then reduced by block diagonalization and truncation. Advanced methods of order reduction have recently been developed by Sofanov (Ref. 10), Meyer (Ref. 11) and Lenz, et al (Ref. 12). The reduced order control law is then optimized in the continuous domain and tested for performance and stability characteristics. This control law is then discretized in a stable manner and is reoptimized in the discrete domain using the analytical gradient expressions which facilitate fast numerical convergence.

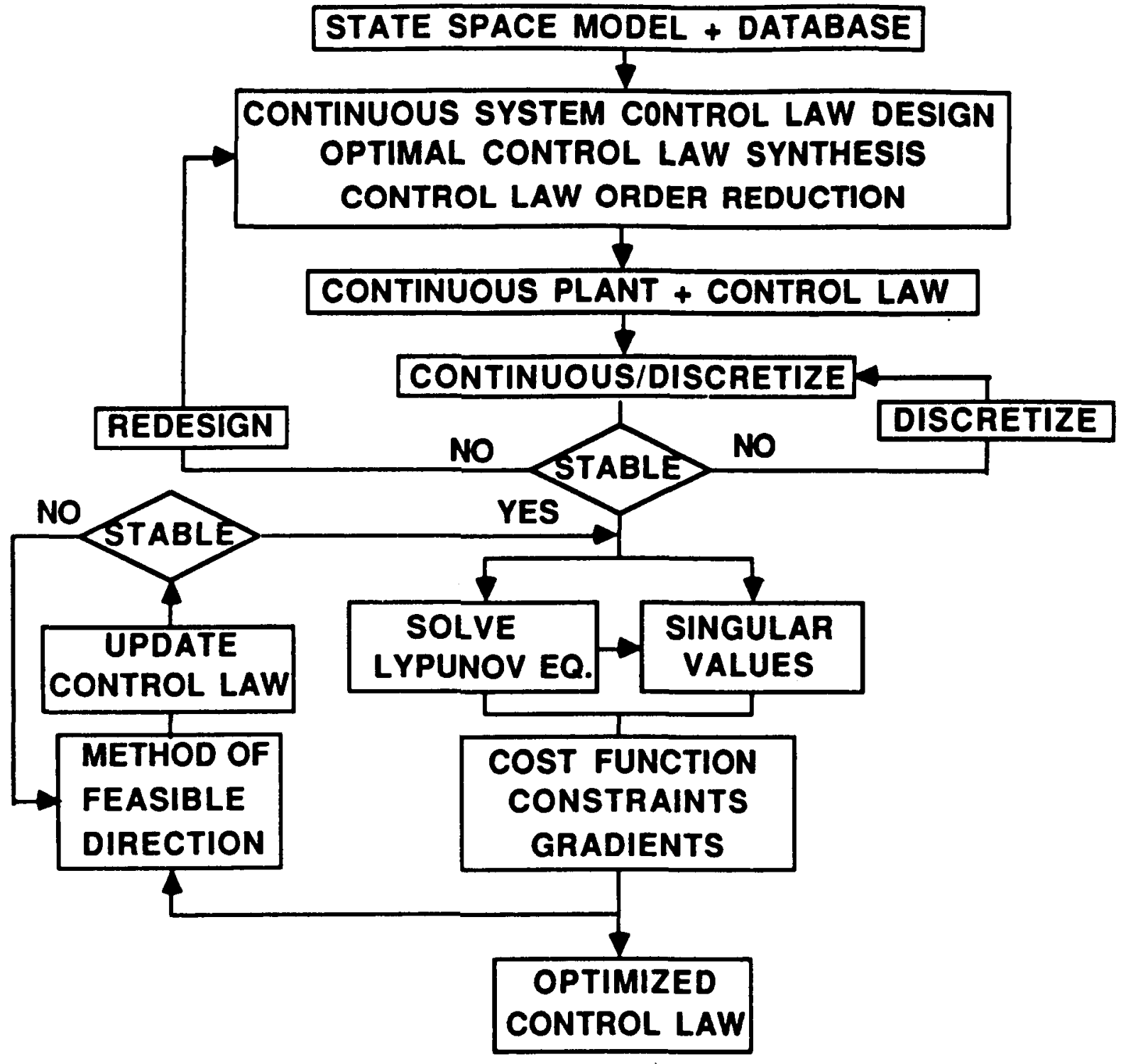

FIGURE 6 


\section{GUST LOAD ALLEVIATION PROBLEM}

A gust load alleviation scheme of a remotely piloted drone aircraft is shown in Fig. 7. The drone is in symmetric longitudinal flight. The random vertical gust is represented by Dryden Spectrum. (Ref. 1). The accelerations are sensed by the fuselage and wing mounted accelerometers and are fedback through a set of antialiasing filters to a digital controller. The sampling rate is 100 $\mathrm{Hz}$. The processed signal activates symmetric deflection command to the elevator and aileron. The primary dynamic loads are generated by the wing flexing due to short period motion. A simple gust load alleviation control law is needed to reduce the open loop RMS bending moment and shear force at the wing root by $50 \%$ without increasing the outboard bending moment and torsion.

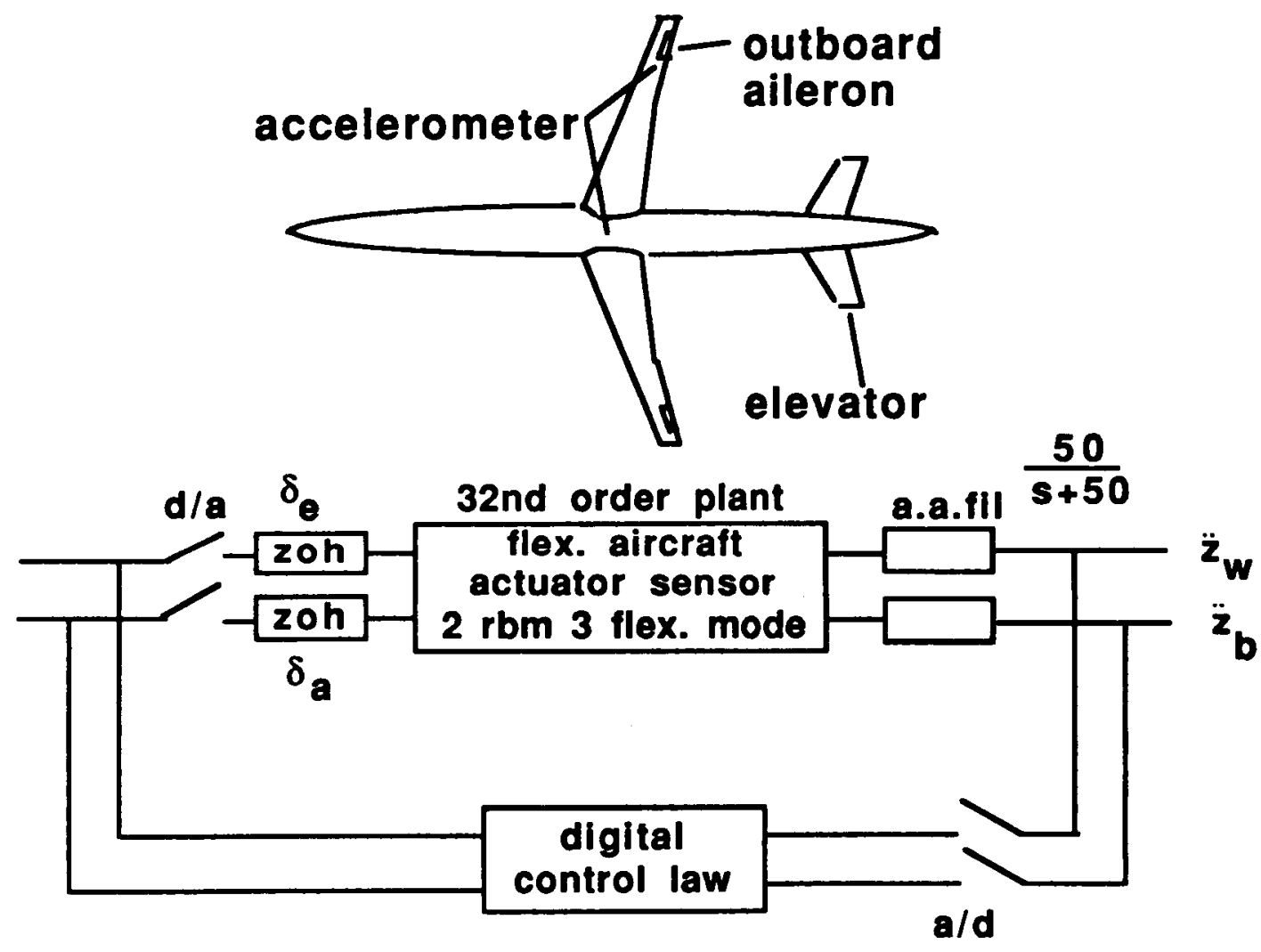

FIGURE 7 


\section{GLA DESIGN OBJECTIVES}

The gust load alleviation (GLA) control law design objectives and synthesis procedure is shown in Fig. 8. The objective is to obtain a low order robust digital GLA control law which would reduce the open loop root-mean- square values of the wing root bending moment and shear by $50 \%$ without increasing the wing outboard bending moment and torsion The control law should maintain certain guaranteed stability margins based on minimum singular value of 0.6 at both the plant input and output (Ref. 3). The control surface deflections and rates should be within the allowable limits. First a full order LQG control law is synthesized to satisfy the design requirements. This 32nd order control law is then reduced to a second order control law and then discretized. This control law does not satisfy the design requirements. After unconstrained optimization most of the requirements are satisfied except the wing outboard bending moment and the singular values. Using constraints on the RMS wing loads and on the minimum singular values of the return difference matrix at the plant input and output, the control law parameters are reoptimized (Ref.2).

\section{GUST LOAD ALLEVIATION DESIGN REQUIREMENTS}
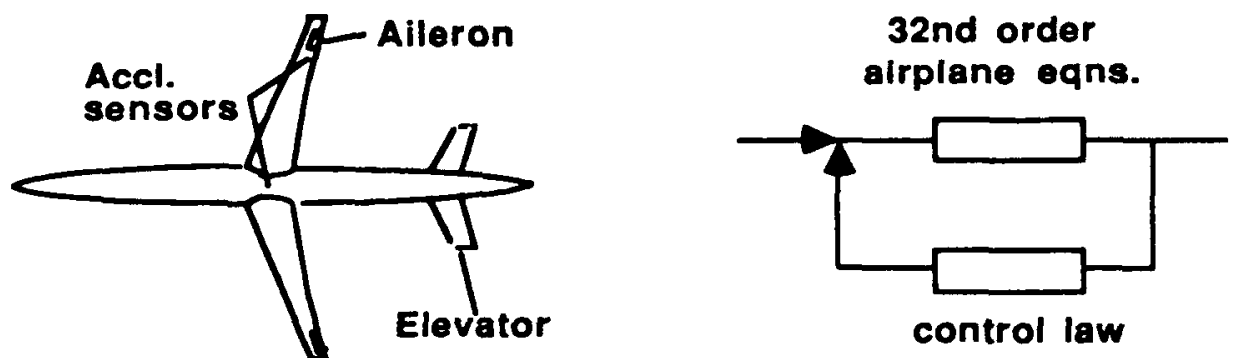

\begin{tabular}{|c|c|c|}
\hline Physical quantities & Design objectives & How we do it \\
\hline Root bending moment & $50 \%$ reduction & \multirow{8}{*}{$\begin{array}{l}\text { 1. LOG design } \\
\text { 2. Control law } \\
\text { order reduction } \\
\text { 3. Discretize } \\
\text { 4. Optimization } \\
\text { 5. Apply constraints } \\
\text { a) on rms loads } \\
\text { b) on singular val. }\end{array}$} \\
\hline Root shear & $50 \%$ reduction & \\
\hline Outboard bending mom & No increase & \\
\hline Outboard torsion & No increase & \\
\hline Elevator deflection & Within max limit & \\
\hline Elevator rate & Within max limit & \\
\hline Aileron deflection & Within max limit & \\
\hline Aileron rate & Within max limit & \\
\hline
\end{tabular}

FIGURE 8 


\section{COMPARISON OF RMS RESPONSES}

The Fig. 9 shows a comparison of RMS responses and control surface deflections for a sequence of second order GLA control laws. The RMS values of wing root bending moment (WRBM), wing root shear (WRS), wing outboard bending moment (WOBM) and wing outboard torsion (WOT) are normalized to their open loop values and control surface deflection and rates are normalized to their maximum allowable values. The control law-I is obtained by digitization of a continuous control law obtained via reduction of a full order LQG design. This control law does not satisfy any of the design requirements. After an unconstrained optimization the control law-II is obtained which satisfies all the RMS response requirements except that on the WOBM. This is satisfied by a constrained optimization sequence to obtain law-III. After imposing the stability margin constraints, the control law -IV is obtained. The stability margins are improved at the cost of increased RMS responses.

\section{COMPARISON OF NORMALIZED RMS RESPONSES DUE TO $1 \mathrm{M} / \mathrm{S}$ RMS DRYDEN GUST}
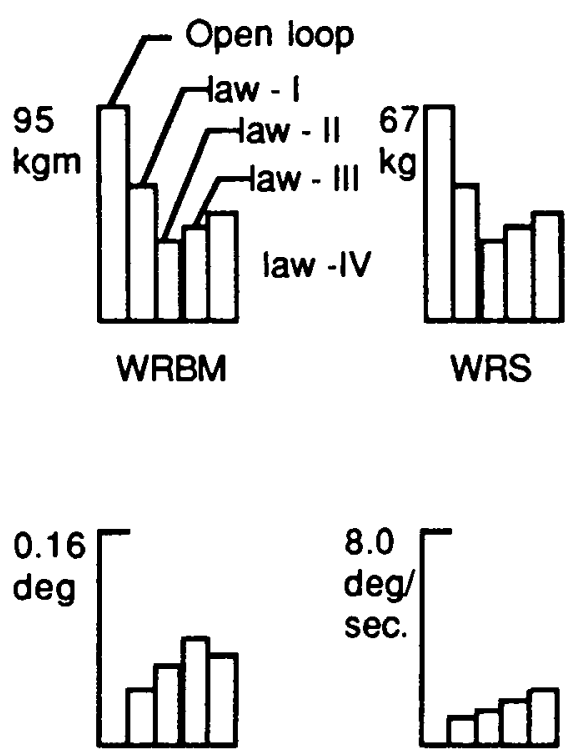

ELEVATOR

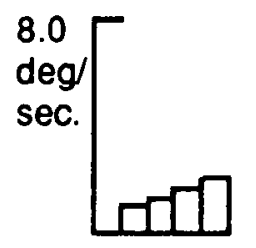

ELV. RATE

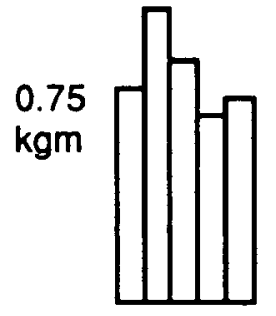

WOBM

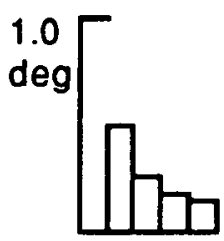

AILERON

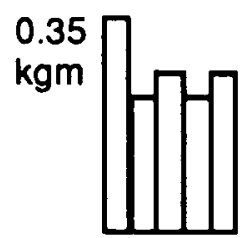

WOT

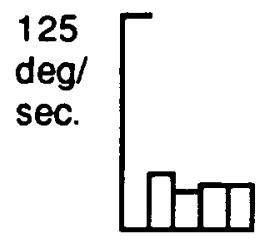

AIL. RATE law - I Initial 2nd order digital law - II After Optimization law - III After Opt. with RMS Constraints law - IV After Opt. with SV Constraints

FIGURE 9 


\section{UNCONSTRAINED AND CONSTRAINED \\ OPTIMIZATION}

The cost function consists of weighted sum of the wing RMS loads and control surface RMS deflections using Bryson's inverse square weighting rule. The plot of normalized cost function versus iteration for the unconstrained optimization process used to obtain law-II is shown in Figure 10. The convergence is obtained in one iteration starting from the initial control law-I. In order to prevent the small increase in the wing outboard bending moment (WOBM), the control law-II is reoptimized by treating WOBM as a constraint instead of lumping it in the cost function. The result of this constrained optimization is also shown in Figure 10. The constraint is satisfied in one iteration, at the expense of increased cost function, which is subsequently reduced along with the wing outboard bending moment.

Unconstrained optimization

Constrained optimization
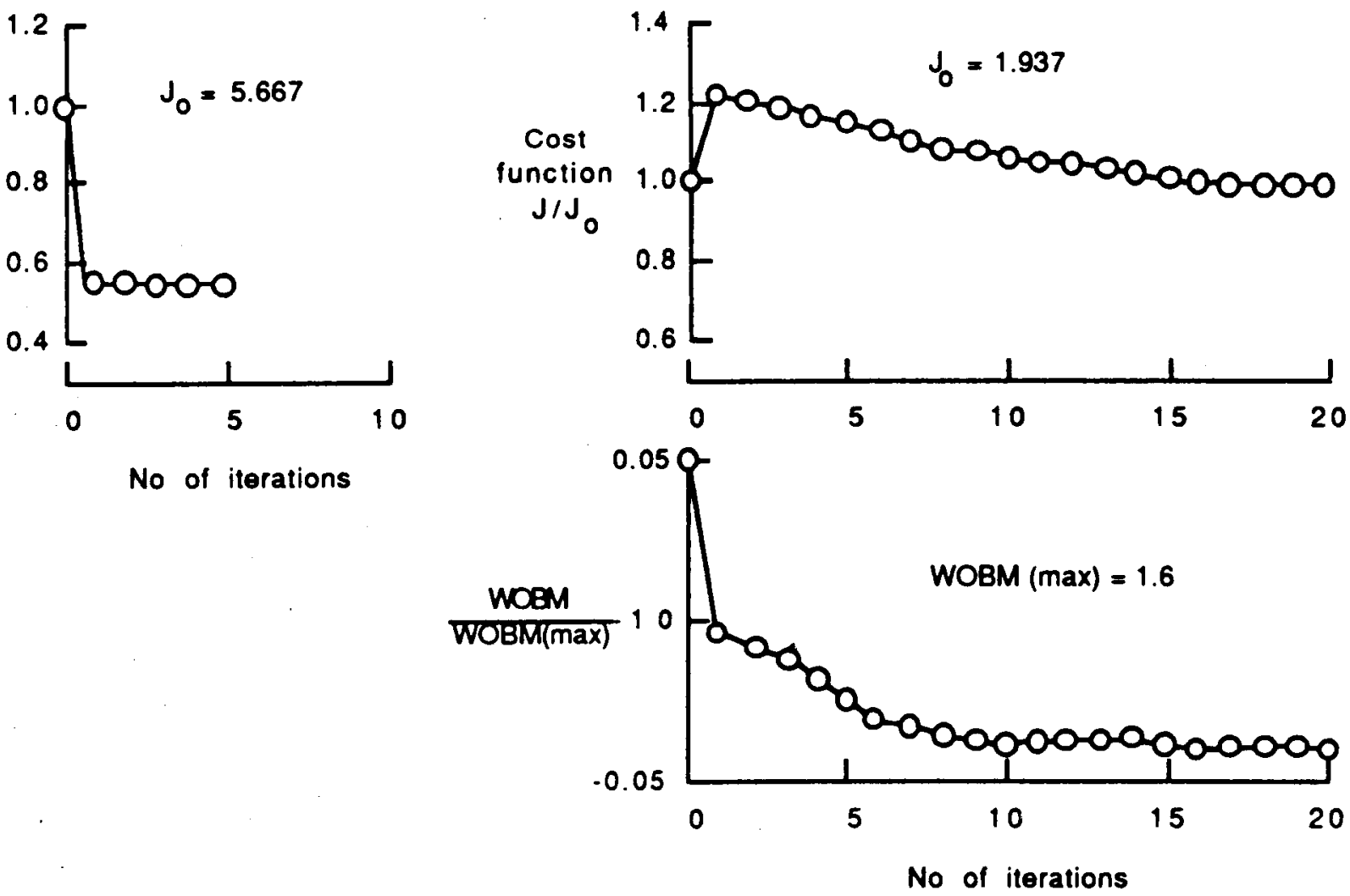

FIGURE 10 


\section{STABILITY ROBUSTNESS}

In obtaining control laws I, II and III, no constraints were applied to the minimum singular value of the return difference matrix at the plant input and output which is a measure of the system's stability robustness properties (Ref. 3 ). The minimum singular value plot of the control laws II and III are shown in Fig. 11 at the plant input and output. In order to maintain a guaranteed phase and gain margins of $+35,-35$ degrees and $+8.0 \mathrm{~dB},-4.0 \mathrm{~dB}$ respectively in each channel, the minimum singular value plots should be above the horizontal dotted line at $-4.43 \mathrm{~dB}$ which corresponds to a minimum singular value of 0.60 . Figure 1 indicates that none of the control laws satisfy these criteria although the control law-III is fairly robust compared to control law -II. Additional constrained optimization is required to improve the stability robustness at the plant input and output. These results are shown next.

At input

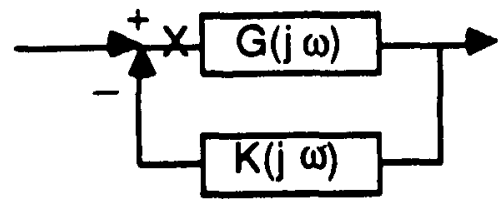

$|a w-1|$

$d B$

$$
10
$$

0

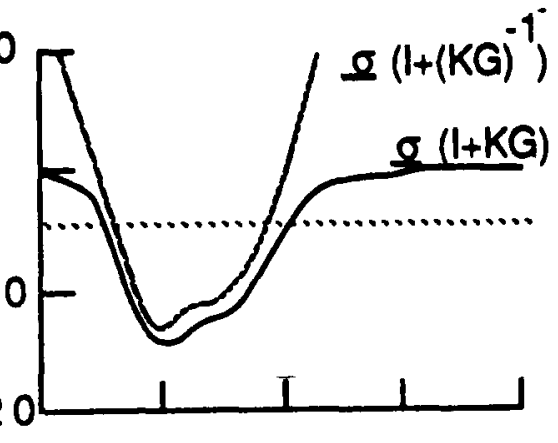

$|a w-11|$

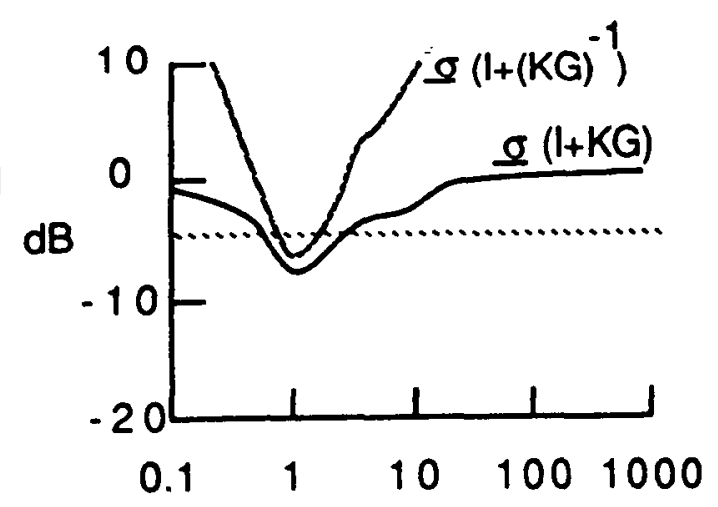

Frequency, rad/s
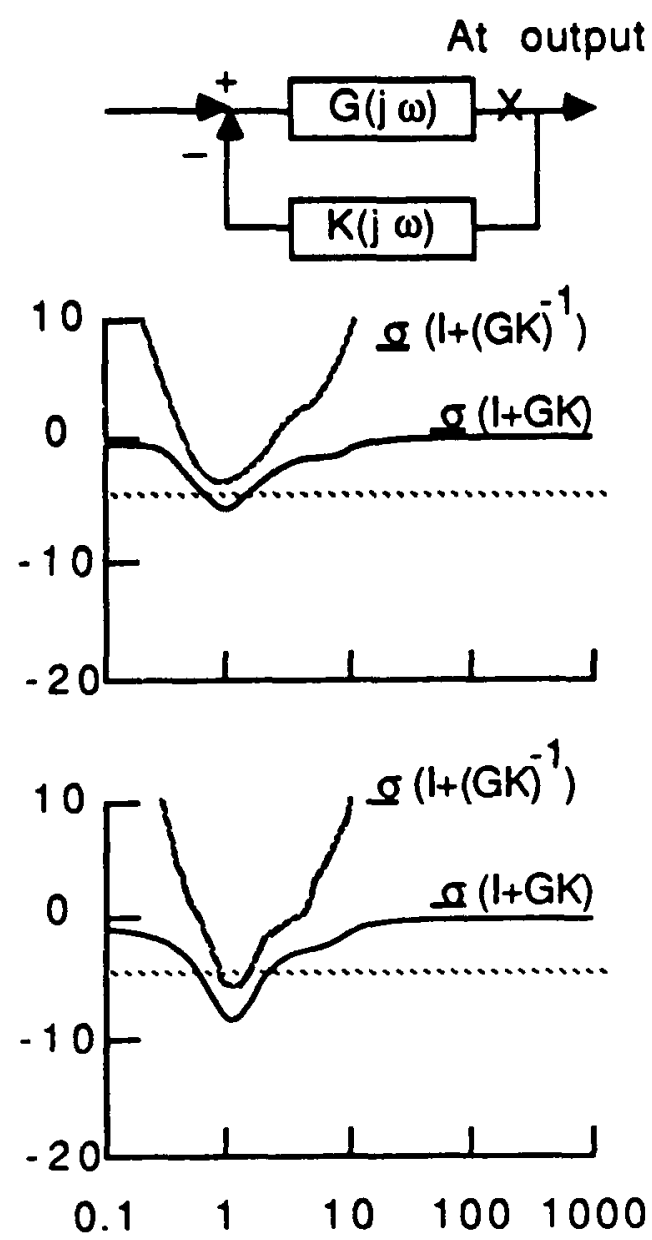

Frequency, rad/s

FIGURE 11 


\section{STABILITY ROBUSTNESS IMPROVEMENT}

In order to improve the stability margins at both the plant input and output, the control law-III was reoptimized using two additional constraints corresponding to the required minimum singular value of $(I+K G)$ and $(I+G K)$ not less than $(0.60)$ or $-4.43 \mathrm{~dB}$. Other constraints were also retained. The resulting control law-IV obtained after 7 iterations satisfies all the constraints. The increased robustness is at the cost of higher RMS responses compared to law-III. The minimum singular value plot is shown in Fig.12. With control law-IV, the system has guaranteed simultaneous gain margins of $+5.7 \mathrm{~dB},-17.0 \mathrm{~dB}$ and phase margins of +53 and -53 degrees at each channel. Thus substantial improvement in stability robustness was obtained by using constrained optimization.
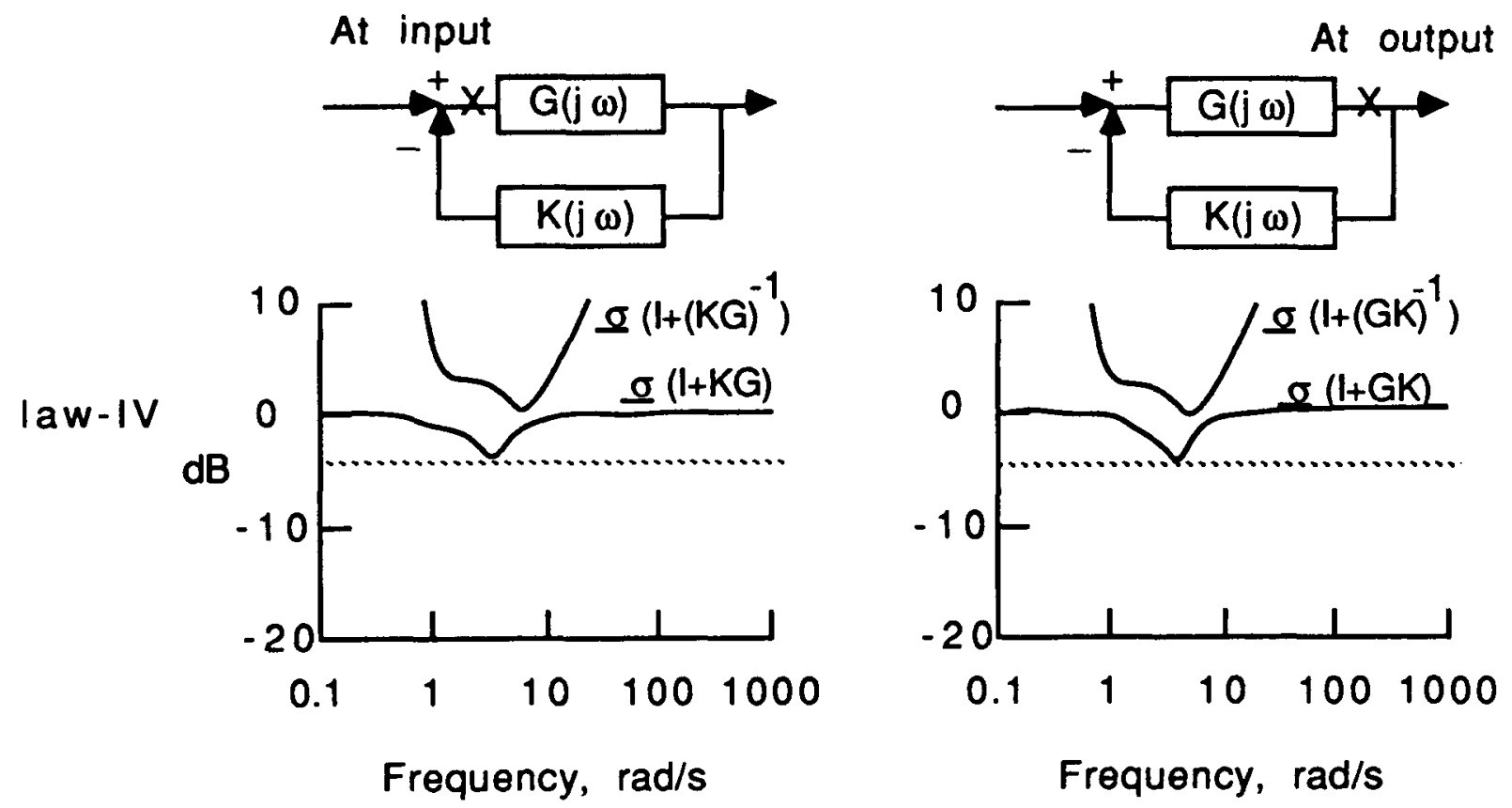

FIGURE 12 


\section{AFW SYMMETRIC FLUTTER SUPPRESSION}

Digital robust control law synthesis for the Active Flexible Wing (AFW) wind tunnel model is presently being carried out in collaboration with Rockwell International. The basic block diagram for a two input two output symmetric flutter suppression system is shown in Fig. 13 for a sting mounted model using leading edge outboard (LEO) and trailing edge outboard (TEO) symmetric actuators and colocated accelerometer sensors. The sampling rate is $200 \mathrm{~Hz}$. The design takes into account the effects of actuator dynamics, 4th order $100 \mathrm{~Hz}$ Butterworth filters and one cycle computational delay at each channel. Full order and reduced order analog and discrete robust control laws were synthesized based on an approximate 38 th order system at 300 psf design dynamic pressure. The discrete 8th order control law was able to stabilize the system over the range 300 to $150 \mathrm{psf}$. The more detailed 80th order model was also stable at 300 and 200 psf. Starting with these preliminary control laws, detailed analysis will be carried out using the discrete system optimization procedure.

\section{SYMMETRIC FLUTTER SUPPRESSION SYSTEM}

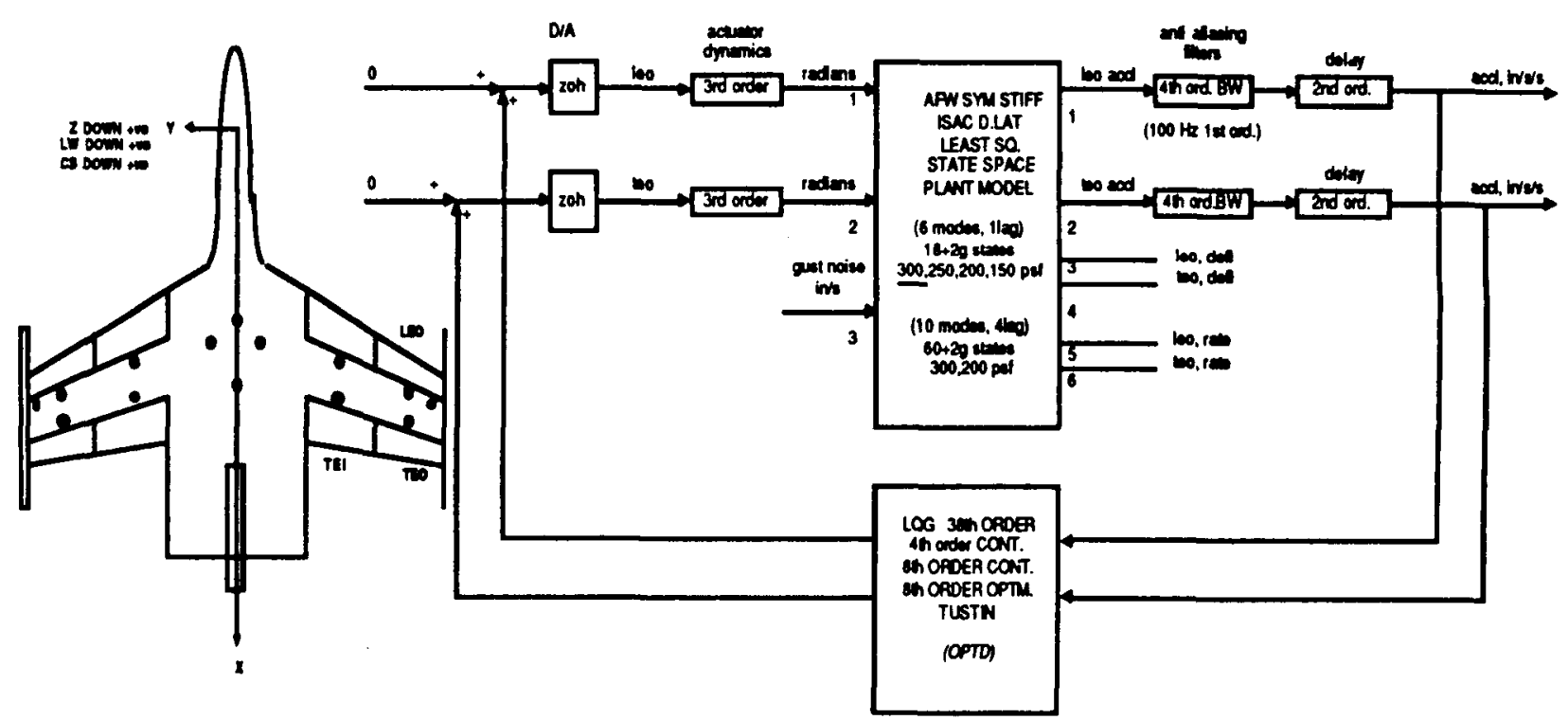

FIGURE 13 


\section{CONCLUSIONS}

Constrained optimization technique was used to synthesize low order robust digital control law for large order flexible systems. The methodology provides a systematic design tool for control system synthesis where a large number of conflicting design requirements on the performance and stability robustness must be satisfied to arrive at a compromise solution. Both continuous and discrete control system can be synthesized and optimized. The procedure can be used to update a classical control law as well as a Kalman estimator based full or reduced order control law. The effects of digitization, antialiasing filters and computational delays can be included in the synthesis process. The synthesis procedure has been successfully applied to a gust load alleviation problem of a drone aircraft and a flutter suppression problem.of the AFW wind tunnel aeroelastic model. Future applications include a rapid roll maneuver load control system design for the AFW wind tunnel model. A block diagram of the control scheme is shown in Figure 14.

Control and vibration suppression of large space structures is another potential application area. The gradient expressions derived to facilitate rapid convergence of the optimization process can also be used for sensitivity study and integrated structure-control optimization formulation.

\section{AFW RAPID ROLL CONTROL}

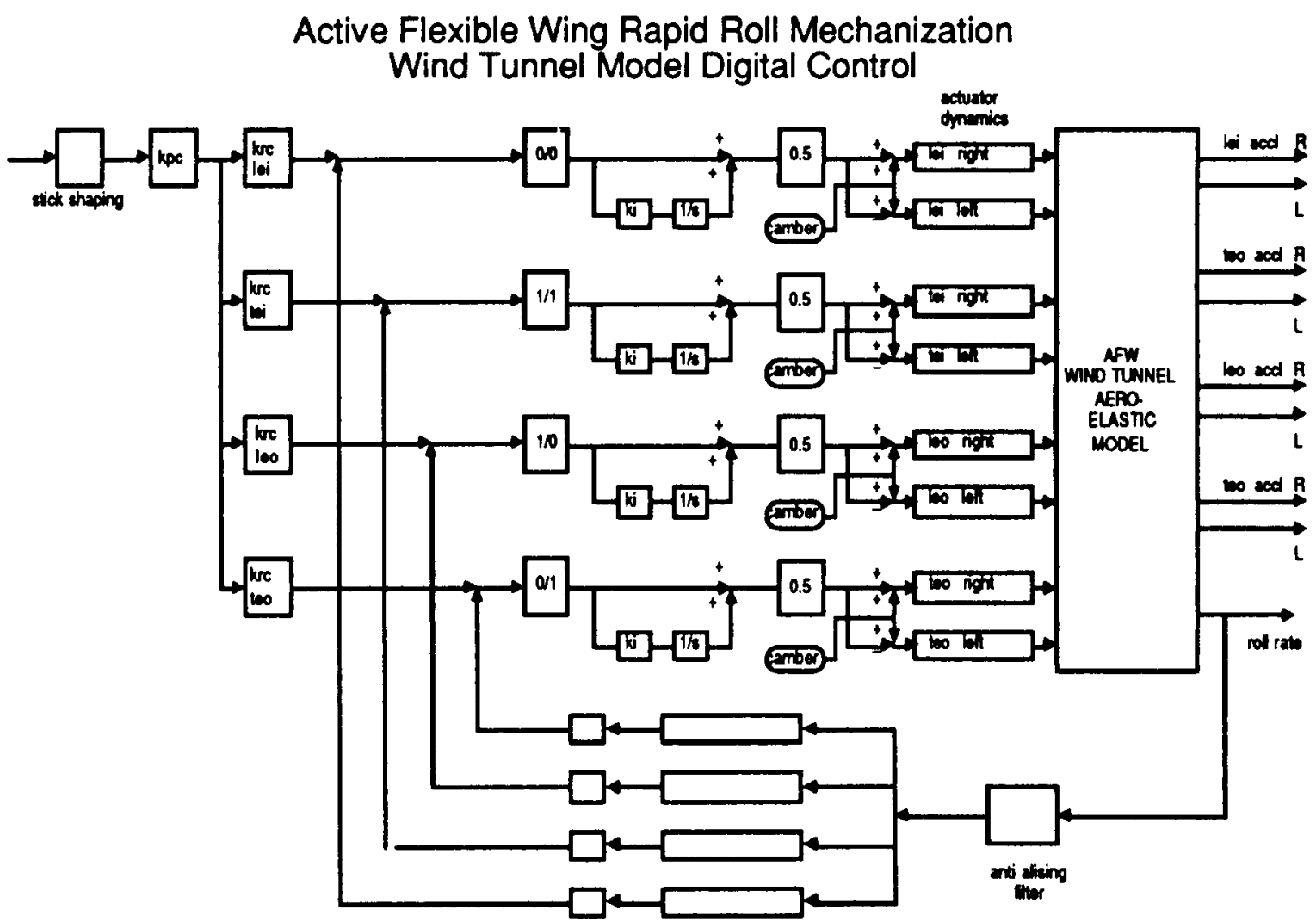

FIGURE 14 


\section{ACKNOWLEDGMENT}

The research support through NASA Langley Contract NAS-180(0) and technical discussion with Irving Abel. Tom Noll, Mike Gilber and Boyd Perry of Structural Dynamics Division and Aeroservoelasticity Branch are gratefully acknowledged.

\section{REFERENCES}

1. Mukhopadhyay, V. , Newsom, J. R. and Abel, I. , "A Method for Obtaining Reduced Order Control Laws for High Order Systems Using Optimization Technique,"NASA TP 1876, August 1981.

2. Mukhopadhyay, V, "Digital Robust Active Control Law Synthesis For Large Order Systems Using Constrained Optimization ," AIAA Paper No. 87-2588, August, 1987.

3. Mukhopadhyay, V., "Stability Robustness Improvement Using Constrained Optimization Techniques," Journal of Guidance, Control and Dynamics, Vol. 10, No. 2, March-April 1987, pp. 172-177.

4. Vanderplatts, G. N.,"CONMIN - A Fortran Program for Constrained Function Minimization - User Manual," NASA TM X 62282, August 1973.

5. Newsom, J. R. and Mukhopadhyay, V., "Application of Constrained Optimization to Active Control of Aeroelastic Response, " NASA TM-83150, June 1981.

6. Martin, G. D. and Bryson, A. E. Jr., "Attitude Control of Flexible Spacecraft," Journal of Guidance, Control, and Dynamics, Vol. 3, pp. 37-41.

7. Gangsaas, D. and Ly, Uy-Loi, "Practical Gust Load Alleviation and Flutter Suppression Control Laws Based on LQG Methodology," AIAA Paper 81- 0021, January 1981.

8. Mukhopadhyay, V., Newsom, J. R. and Abel, I., "Reduced Order Optimal Feedback Control Law Synthesis for Flutter Suppression," Journal of Guidance, Control, and Dynamics, Vol. 5, July-Aug. 1982, pp. 389-395.

9. Uy, Uy-Loi, "A Design Algorithm for Robust Low Order Controllers,"Department of Aeronautics and Astronautics, Stānford University, SUDAAR Report No. 536, Nov.1982.

10. Safonov, M. G. and Chiang, Y. A.," A Schur Method for Balanced Model Reduction," Proc. of 1988 American Control Conference, Atlanta, Ga., June, 1988, pp. 1036-1040.

11. Meyer, D. G.,, "A fractional Approach to Model Reduction," Proc. of 1988 American Control Conference, Atlanta, Ga, June, 1988, pp. 1041-1047.

12. Lenz, K. E., P. Khargonekar and Doyle, J. C.,"Controller Order Reduction with Guaranteed Stability and Performance," Proc. of 1988 ACC, June 1988, pp. 1697-1698. 